\title{
Comparison of PMD Compensation in WDM Systems
}

\author{
Anju Kochumman ${ }^{1}$, Lakshmy G.B. ${ }^{2}$ \\ ${ }^{I}$ (PG Scholar, Dept. of Electronics \& Communication Engg., TKM Institute of Technology, Kerala, India) \\ ${ }^{2}$ (Asst.Professor, Dept. of Electronics \& Communication Engg., TKM Institute of Technology, Kerala, India)
}

\begin{abstract}
The need for larger capacities in long haul optical digital transmission lead to greater channel density which can be achieved by wavelength division multiplexing and increasing the bit rate of each channel. As data rates increases, certain phenomena such as dispersion began to show up as obstacles. At higher bit rates beyond 2.5Gbps polarization mode dispersion (PMD) becomes a main factor in the degradation of the transmission characteristics. PMD occurs when slightly different planes of light inside a fiber travel at slightly different speeds and make it impossible to transmit data reliably at high speed in single mode fibers. PMD is caused due to optical birefringence in the fiber due to which the two modes within a single mode fiber travel with different group velocities and the random change of this birefringence along the fiber length results in random coupling between the modes. This effect of PMD results in broadening of transmitted pulses that limit the transmission capacity of the fiber. In high-speed optical communication systems working at data rates of 10 Gbps and beyond, signal distortion caused by PMD is also a major limitation of the transmission distance. This paper intends to analyze the performance of PMD compensation by optical compensation technique and using DCF in a two channel WDM system. The analysis is done through eye diagrams from which the $Q$ value and bit error rate can be determined by simulating with OptSim5.3, which includes the latest simulation algorithms to guarantee the highest possible accuracy and real world results.
\end{abstract}

Keywords- Birefringence, Data rate, Deterministic Differential Group Delay (DDGD), Dispersion compensating fibers (DCFs) Polarization Mode Dispersion (PMD Principal State of Polarization (PSP), $Q$ value.

\section{INTRODUCTION}

Optical fiber communication plays a vital role in the development of high quality and high-speed telecommunication systems. Fiber optic communications is a way of exchanging the information between two places by sending the light signal through the optical fiber cable. Fiber optic communication brought the revolutionary change in the telecommunication industry and played a major role in the advent of information age. In the twenty first century, its advantages over electrical transmission cause the replacement of copper wire with the optical fiber in the communication system. Now the optical fiber is the most common type of channel used in communication system, but the other types of waveguides are also used within the communication system. Today, optical fibers are not only used in telecommunication links but also used in the Internet and local area networks (LAN) to achieve high signaling rates. The core advantages of optical fiber such as low loss, which allows long distances between amplifiers and its high data carrying capacity as that of thousands of electrical links would be required to carry that much data. Also no crosstalk introduces in optical fibers running alongside each other for long distances as introduces in some types of electrical transmission lines.

The needs for larger capacities in long haul optical transmission lead to greater channel density achieved by wavelength division multiplexing and increasing the bit rate for each channel. As the number of channels and data rates increases, certain phenomena such as dispersion began to show up as obstacles. The optical fiber has some inherent properties like birefringence, which leads to what is called polarization mode dispersion (PMD). PMD effects are linear electromagnetic propagation phenomena occurring in so-called "single-mode" fibers. Despite their name, these fibers support two modes of propagation distinguished by their polarization. Because of optical birefringence in the fiber, the two modes travel with different group velocities and the random change of this birefringence along the fiber length results in random coupling between the modes. The resulting PMD phenomena lead to pulse distortion and system impairments that limit the transmission capacity of the fiber.

Polarization Mode Dispersion which is a time varying quantity is a serious problem that limits distances and data rates in a single-mode optical fiber system. In high-speed optical communication systems working at data rates of $10 \mathrm{Gbps}$ and beyond, signal distortion caused by polarization mode dispersion (PMD) is a major limitation of the transmission distance. This leads to degradation in system performance. Especially in upgrading of existing transmission lines, high PMD of 'old' fibers is a serious problem. The PMD of installed fibers fluctuates with time due to environmental influences, for example changes in temperature and stress. Therefore, adaptive PMD compensation during system operation is indispensable. 
In general, the effect of PMD on an optical fiber communication link at different bit rates shows that it causes several undesirable effects and it can be compensated through various compensation techniques. The goal of this work is to analyze the effect Polarization Mode Dispersion compensation in a two channel WDM system.

Here the effect of PMD compensation method is also proposed by which the degradation due to PMD can be mitigated to a certain extent. The basic principle of optical compensation method is to split the optical signal that exits from the optical fiber into two branches and sent to two polarizers which are complementary to each other to obtain two polarization states. A suitable delay is provided to one of the polarization states and no delay to the other state. Finally the two signals are added and as a result the two components experience a differential delay. This differential delay cancels the differential group delay caused due to the effect of PMD. Also the effect PMD compensation using dispersion compensating fibers in the post compensation mode is also analyzed.

\section{SYSTEM MODELING}

Polarization mode dispersion is a physical phenomenon in optical fibers that causes light pulses to spread in time. In high speed transmission systems beyond 2.5 Gbps increase in PMD causes the broadening of pulses and hence adjacent pulses overlap and interfere with each other causing intersymbol interference (ISI).Thus the effect of PMD severely degrades the system performance. The cause of PMD is birefringence which can be due to geometrical variations in concentricity or ellipticity of fiber core or cladding during fiber manufacture or due to stress on fiber because of pressure or temperature variations. Thus PMD is varying randomly along the fiber length and therefore the effect of PMD can be reduced by introducing better fiber manufacturing techniques or by compensating for PMD effects. Compensation can be achieved by electronic equalization or by using dispersion compensating fibers or by optical equalization. Electronic compensation is not sensitive to the phase and polarization information of the received signal and also it is bit rate dependent so optical compensation is advantageous compared to electronic compensation. Therefore in this work an optical compensation method is proposed to compensate for the effect of PMD.

\subsection{Basic Block Diagram}

The block diagram of the work is shown in the Figure.1and 2. It describes the basic components used for the analysis of the effect of PMD compensation.

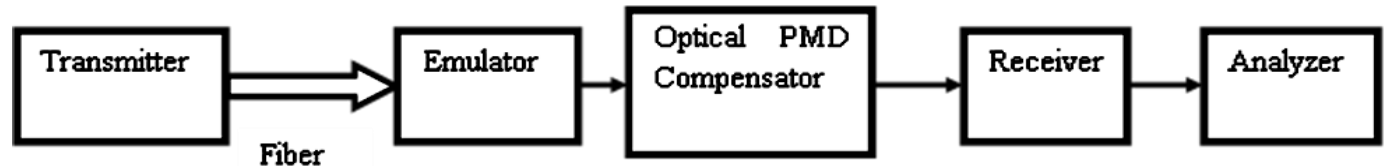

Figure 1: Block diagram for optical PMD compensation

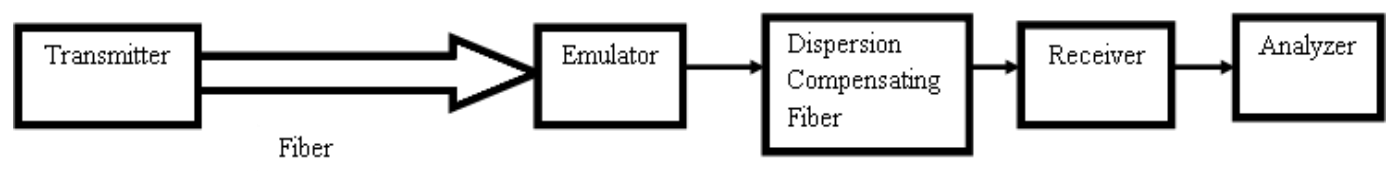

Figure 2: Block diagram for PMD compensation using DCFs

In Figure 1 the optical compensation technique of using an emulator and a deterministic differential group delay is analyzed. An emulator is used after the fiber link to recreate the behavior of an optical field fiber in the laboratory. In Figure 2 the compensation using DCF is analyzed. Depending upon the position of placing the DCF it can be of two types-pre compensation and post compensation. In pre-compensation the DCF is placed before the fiber and in post-compensation technique the DCF is placed after the fiber. In this paper the post-compensation technique is analyzed which is more efficient than the pre-compensation technique.

\subsection{Simulation Block Diagram}

The simulation is done in OptSim which is one of the costliest simulation software used for optical communication. The below setup shown in Figure 3 simulates PMD effects with and without compensation. The compensation is done by using a Deterministic Differential Group Delay. The effect can be analyzed through eye diagrams and Q estimator. 


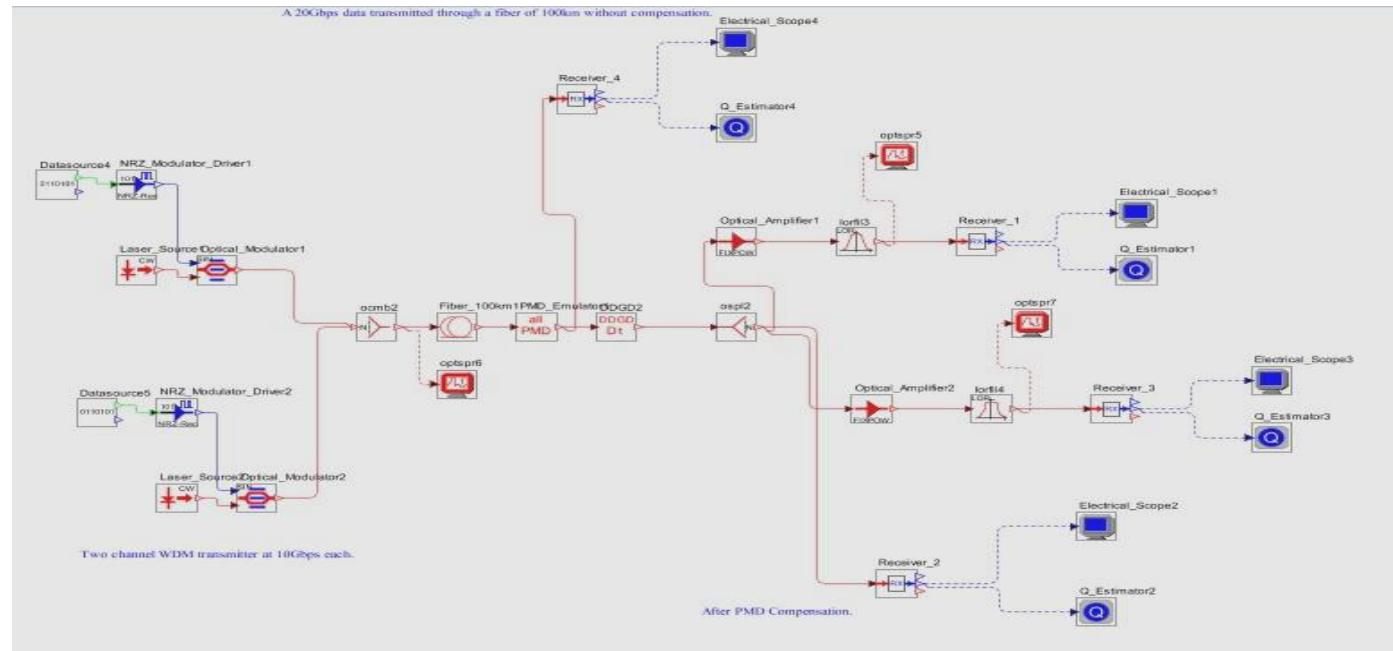

Figure 3: OptSim layout to analyze PMD compensation in two channel WDM using optical compensation

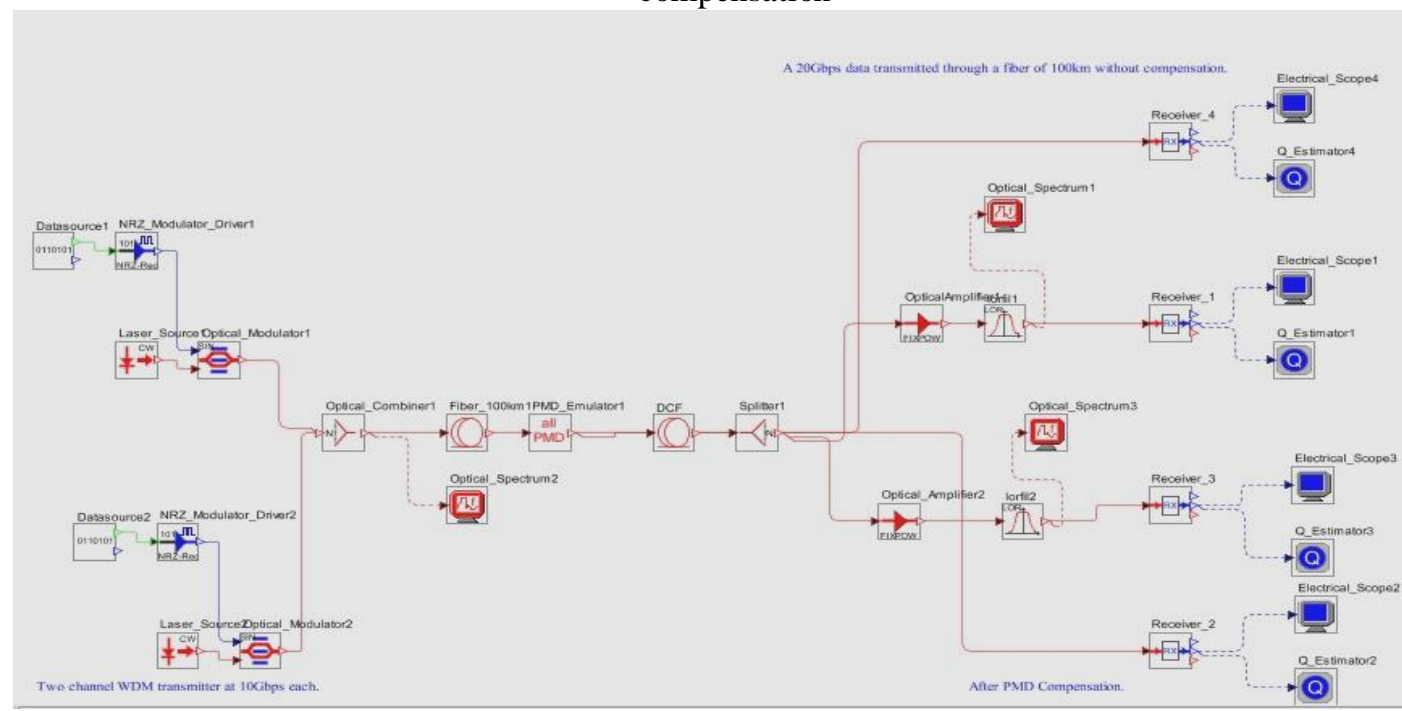

Figure 4: OptSim layout to analyze PMD compensation in two channel WDM using post-DCF

Above figures shows a two wavelength WDM system capable of transmitting 20Gbps data consisting of two transmitters and two receivers. The transmitters and receivers sections are connected by the dispersive fiber link. The transmitter section consists of data source, modulator driver, laser source and modulator. Each data source produces a pseudorandom sequence of bits at a rate of $10 \mathrm{Gbps}$. So a $20 \mathrm{Gbps}$ data can be send a time with two sources along the single fiber channel The output of data source is given to modulator driver which produces a NRZ (Non return to zero) format pulse train. The transmitted signal is formed by modulating the light carrier by the NRZ data source. The light carrier is generated by Lorentzian laser source at the 1548 and $1552 \mathrm{~nm}$ wavelength. The light can be modulated by the input signal by passing through the amplitude modulator. Amplitude modulator modulates the signal. Then the signal is passed through the fiber channel of $100 \mathrm{~km}$. As the signal travels through the fiber the effect of PMD affects the transmitted signal. For laboratory setup in order to recreate the time varying effect of PMD an emulator is used. The emulator rotates the state of polarization which in turn produces the differential group delay and hence the pulse broadens and signal degrades. The distorted signal is received by the receiver and is analyzed through eye diagrams. For analyzing the optical compensation shown in Figure 3, the signal emulated by the emulator is passed through a deterministic differential group delay element which splits the signal into two and provides a determined delay to one of the signal. This delay provided negates the distorted signal through the emulator and thus compensation of PMD is achieved which can be seen from the eye diagrams. Figure 4 shows the OptSim layout for analyzing the PMD compensation using post-DCF, where a suitable length of DCF is selected based on the design rule equation:

$$
L_{\text {fiver }} D_{\text {fíbr }}+L_{D C F} D_{D C F}=0
$$


where $L_{f i b s}, D_{f i b s}, L_{D C F}, D_{D C F}$ are the length and dispersion coefficient of the fiber and DCF respectively.

\section{Simulation ReSUlts}

The effect of PMD compensation using an optical compensation technique and dispersion compensating fiber in a $\mathrm{WD} \mid \mathrm{M}$ is simulated using OptSim 5.3. The effect is analyzed through various eye diagrams by performing parametric run. A parametric run is performed to simulate the time varying property of the emulator. The eye diagrams give the $\mathrm{Q}$ values at each run from which the results can be interpreted. The results of the simulations are shown below.

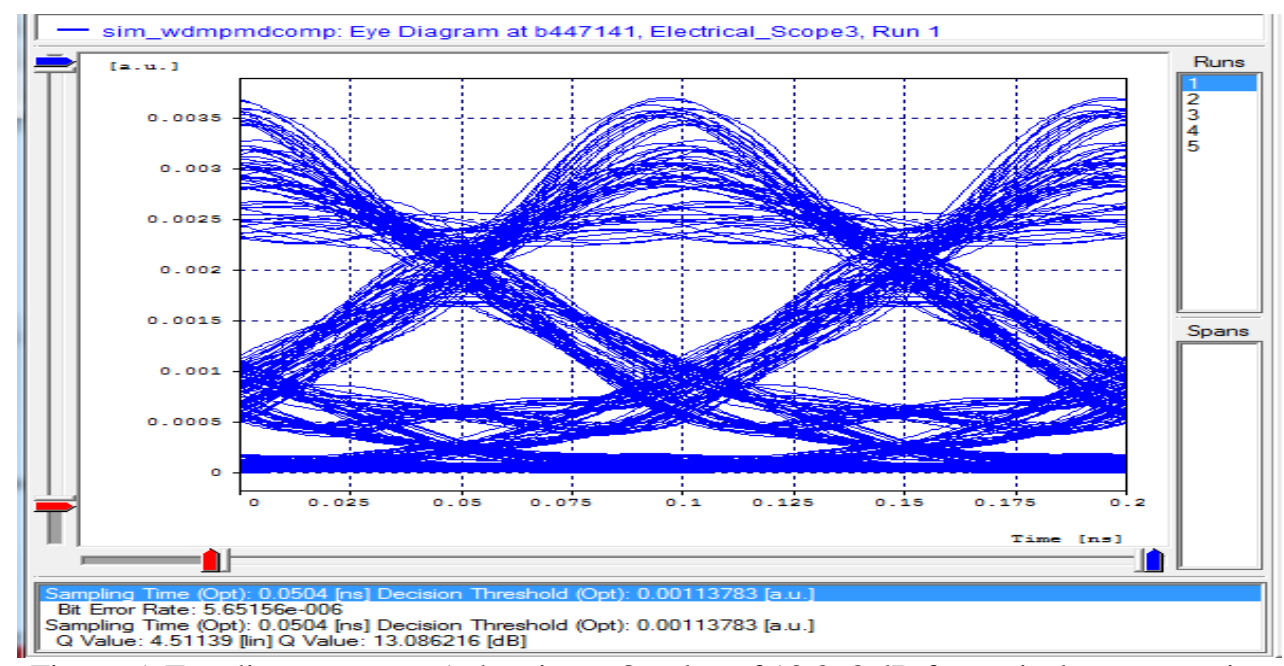

Figure 5: Eye diagram at run 1 showing a $\mathrm{Q}$ value of $13.068 \mathrm{~dB}$ for optical compensation

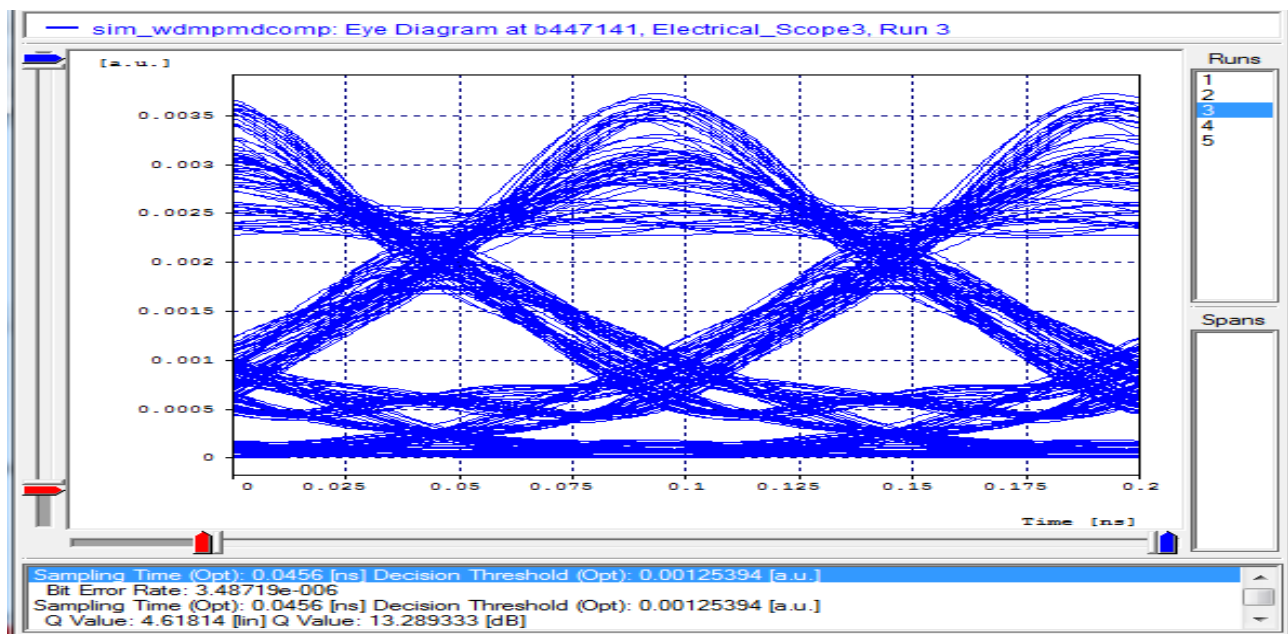

Figure 6: Eye diagram at run 3 showing a $Q$ value of $13.2893 \mathrm{~dB}$ for optical compensation

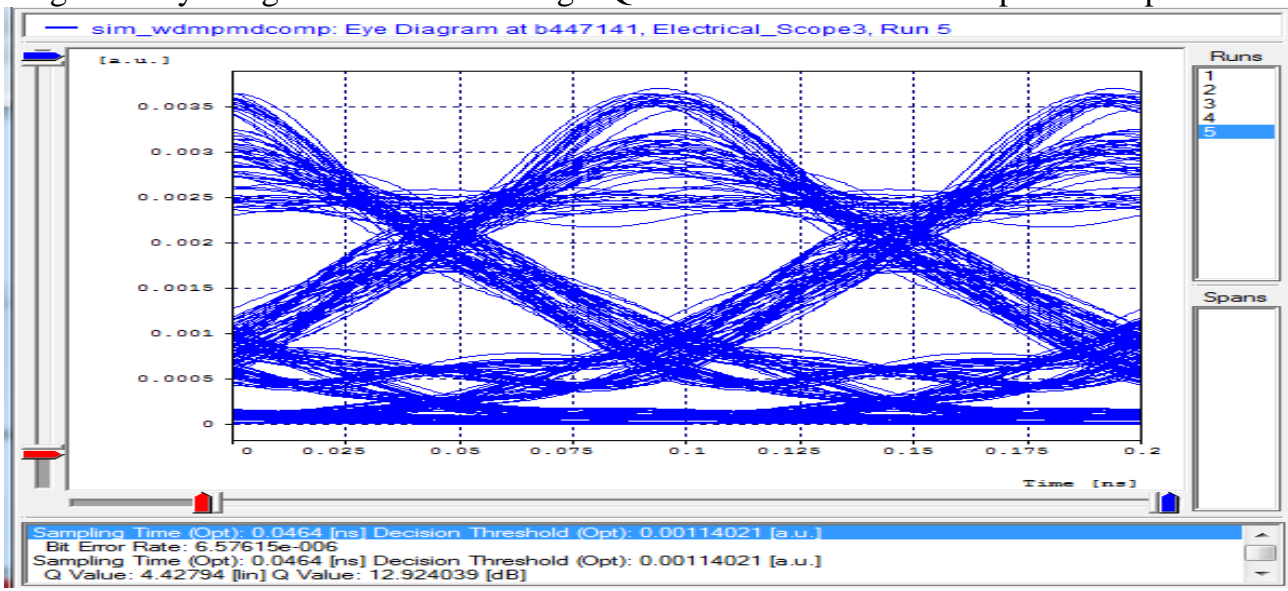

Figure 7: Eye diagram at run 5 showing a $\mathrm{Q}$ value of $12.924 \mathrm{~dB}$ for optical compensation 


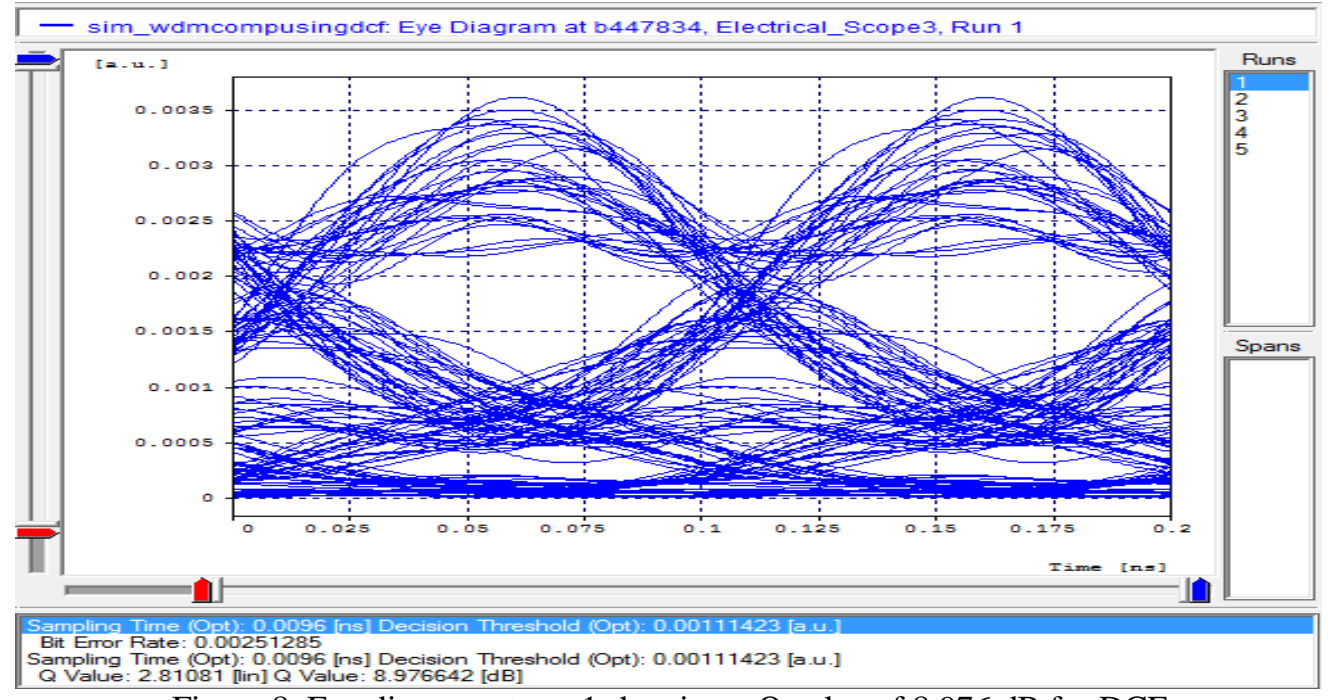

Figure 8: Eye diagram at run 1 showing a $\mathrm{Q}$ value of $8.976 \mathrm{~dB}$ for DCF

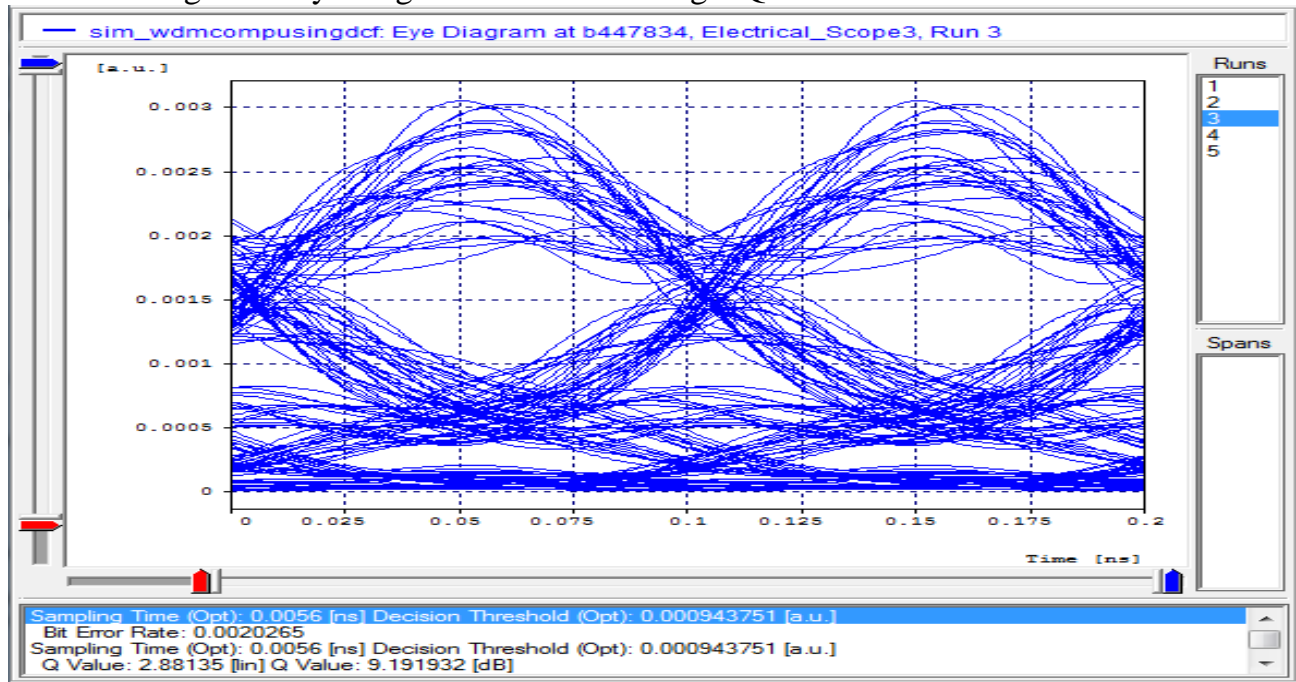

Figure 9: Eye diagram at run 3 showing a $Q$ value of $9.191 \mathrm{~dB}$ for DCF

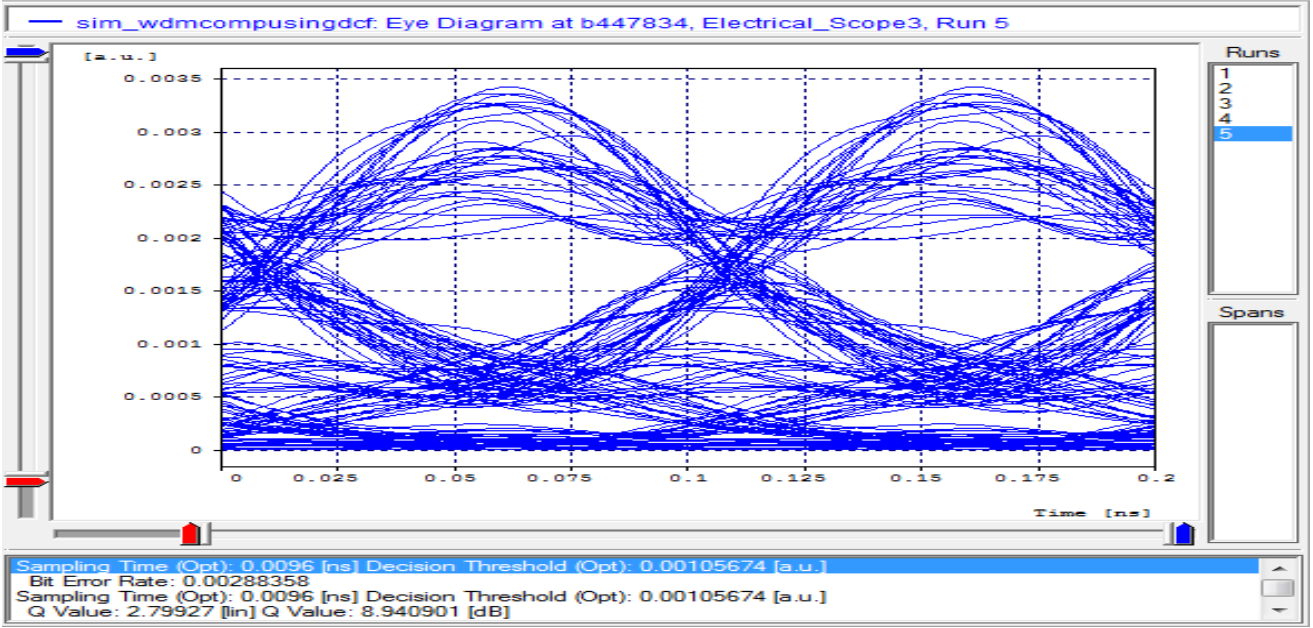

Figure 10: Eye diagram at run 5 showing a $Q$ value of $8.940 \mathrm{~dB}$ for DCF

Figures 5 to 7 shows the effect of optical compensation and Figures 8 to 10 shows the effect of compensation using post-DCF detected at one of the receivers. The two techniques can be compared by analyzing the $\mathrm{Q}$ values of the obtained results which shows a higher $\mathrm{Q}$ value for optical compensation technique. figure below.

The comparison of the optical compensation technique and that using post-DCF is outlined in the 


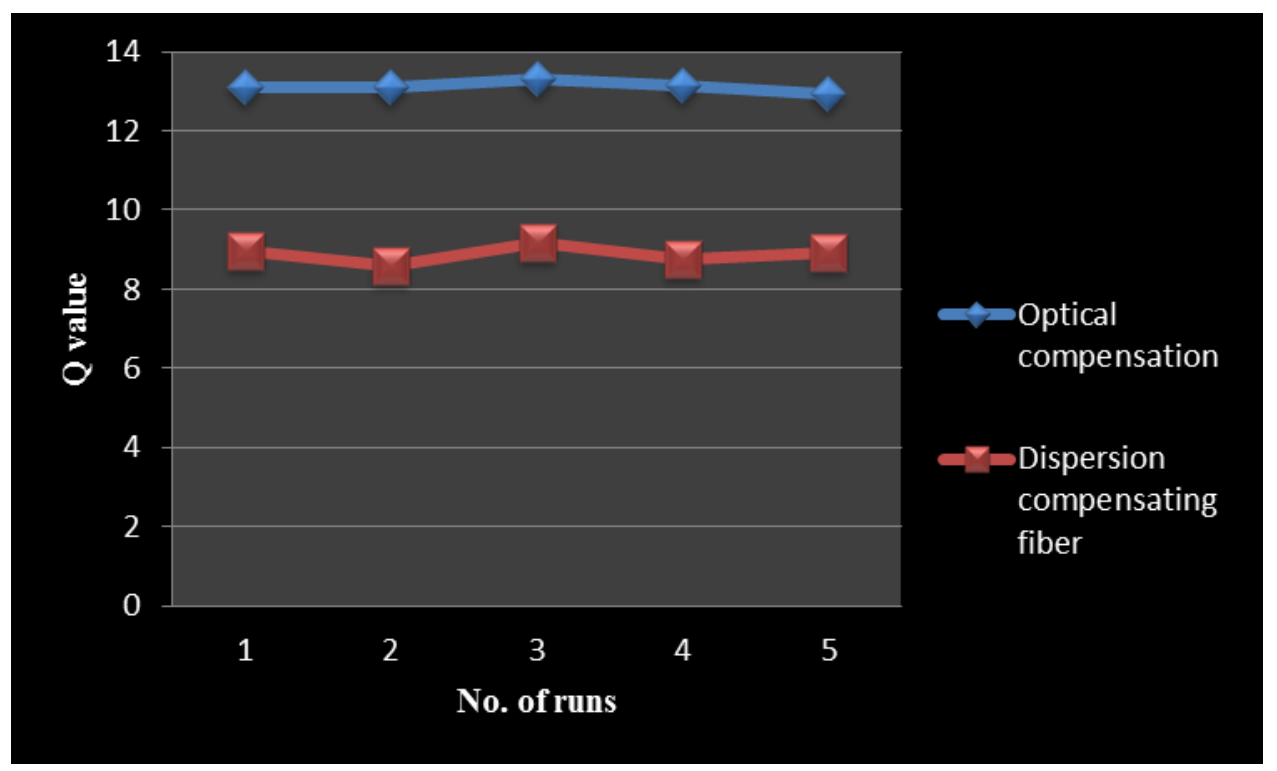

Figure 11: comparison of PMD compensation using optical compensation and DCF

\section{CONCLUSION}

The paper is based on the performance analysis of an optical compensation of polarization mode dispersion which is a time varying quantity which causes a serious problem that limits distances and data rates in a single-mode optical fiber system. The time varying property of polarization mode dispersion in a fiber link is analyzed through various eye diagrams by parametric run in OptSim simulation. An optical compensation method by which the effect of PMD can be mitigated is shown as an increase in Q value and decrease in BER from the eye diagram of the simulation. The results show that optical compensation technique is suitable for PMD compensation in WDM systems compared to dispersion compensating fibers.

\section{REFERENCES}

[1] Krzysztof Borzycki, "Temperature dependence of polarization mode dispersion in tight-buffered optical fibers", Journal of Telecommunications and Information Technology, 2008.

[2] A.M. Agarkar, PrajaktaJoharapurkar, "PMD \& DGD Performance Analysis in SMF due to Fiber Irregularities", International Journal of Computer Applications, Volume 12- No.6, December 2010

[3] David S. Waddy, Liang Chen, XiaoyiBao, “A Novel Dynamical Polarization Mode Dispersion Emulator”,IEEE.

[4] GildasChauvel - Anritsu Corporation, Dispersion in Optical Fiber, 2008.

[5] Kapil Kashyap, Hardeep Singh, "Study the effects and compensation of polarization mode dispersion at different bit rates", IOSR Journal of Engineering, 2012.

[6] Henning Bülow, Chongjin Xie, Axel Klekamp, Xiang Liu,and Bernd Franz "PMD Compensation/Mitigation Techniques for HighSpeed Optical Transport”, Published by Wiley Periodicals,Bell Labs Technical Journal 14(1), 105-124, 2009.

[7] Md Zaini Jamaludin, Ahmad Fauzi Abas, Ahmad Shukri Muhammad Noor and Mohamad Khazani Abdullah, "Issues on polarization mode dispersion (PMD) for high speed fiber optics transmission”, Suranaree J. Sci. Technol.12 (2).

[8] R.Gowri Manohari, T.Sabapathi, "Analysis and reduction of polarization mode dispersionin an optical fiber", International Conference on Recent Advancements in Electrical, Electronics and Control Engineering, 2011.

[9] Kogelnik, H., R.M. Jopson and L.E.Nelson, "Polarization-Mode Dispersion", Chapter15, Optical Fiber Telecommunications, volume IV B, edited by I.P.Kaminow and T.Li, Academic Press, San Diego, CA, 2002. 